\begin{tabular}{|c|c|c|}
\hline & Int.J.Curr.Microbiol.App.Sci (2016) 5(10): 1030-1038 & \\
\hline & International Journal of Current Microbiology and Applied Sciences & \\
\hline & ISSN: 2319-7706 Volume 5 Number 10 (2016) pp. 1030-1038 & \\
\hline $\begin{array}{l}\text { EXCELLENT } \\
\text { PUBLISHERS }\end{array}$ & Journal homepage: http://www.1jcmas.com & \\
\hline PUBLISHERS & & wwwi.jicmas. \\
\hline
\end{tabular}

Original Research Article

http://dx.doi.org/10.20546/ijcmas.2016.510.109

\title{
Epidemiology of Streptococcus pyogenes in Pyogenic Infections in Gulbarga, India
}

\author{
Sanjay Rathod $^{1 *}$, Muzaheed ${ }^{2}$, Venkat M. Shinde ${ }^{3}$ and H.P. Jai Shanker Pillai ${ }^{1}$ \\ ${ }^{1}$ Department of Post Graduate Studies and Research in Microbiology, \\ Gulbarga University, Kalaburagi, India - 585106 \\ ${ }^{2}$ Department of Clinical laboratory Sciences, College of Applied Medical Sciences, \\ University of Dammam, P.O.2435, Dammam 31441 \\ ${ }^{3}$ Department of Post Graduate Studies and Research in Botany, Gulbarga University, \\ Kalaburagi, India - 585106 \\ *Corresponding author: Sanjaymotilal77@gmail.com
}

\section{Keywords}

Streptococcus

pyogenes,

Phayngitis,

multiple

resistances.

Blood agar,

Bacitracin,

penicillin.

\begin{tabular}{l}
\hline Article Info \\
\hline Accepted: \\
30 August 2016 \\
Available Online: \\
10 October 2016
\end{tabular}

\section{A B S T R A C T}

To study the prevalence of Streptococcus pyogenes in general population of different ages, and strata of socio-economic groups. This was a cross-sectional prospective study carried on the general population of poor socio-economic status over one year, by procuring data from the total 129 patient records of OPD patients. The clinical settings including history, examination and the (throat swab, pus cervical swab, sputum ear-discharge cultures) with anti-microbial sensitivity testing performed under standard conditions. In this study, Streptococcus pyogenes was isolated, of Out of the 129 samples screened, S. pyogenes was isolated from 51 samples indicating that $39.53 \%$ of pyogenic infections are being caused by $S$. pyogenes The incidence of $S$. pyogenes was found to be very high among males than females. Out of the 89 samples collected from males, 43 yielded $S$. pyogenes, indicating an incidence of $48.31 \%$. While in the case of females out of 40 samples only 8 samples showed the presence of $S$. pyogenes indicating an incidence of only $20 \%$. The incidence was also high in pus $(50 \%)$ and throat swab (48\%) samples. While the same was very low in cervical swabs (10\%) and ear discharge (11\%). The antibiogram pattern indicated an alarming situation, in that among " $S$. pyogenes isolates were resistant to multiple antibiotics all the 51 isolates were resistant atleast to three antibiotics. The multiple resistances ranged from 3 to 8 antibiotics. Among the 51 isolates, as many as 17 (33\%) were resistant to 5 antibiotics and $14(27 \%)$ to 6 six antibiotics respectively. The study showed S.Pyogenes and also showed significant amount of resistance against commonly used antibiotics which were earlier sensitive probably due to injudicious use of antibiotics without undergoing routine culture and sensitivity.

\section{Introduction}

Streptococcus pyogenes has persisted and is now resurging as an important hospital and community acquired pathogen. Streptococcus pyogenes is one of the most 
frequent pathogens of humans. It is estimated that between $5-15 \%$ of normal individuals harbor the bacterium, usually in the respiratory tract, without signs of disease. S. pyogenes is the most pathogenic bacterium in the genus Streptococcus; it is the agent that causes bacterial pharyngitis, impetigo, scarlet fever, rheumatic fever and a host of other infections including severe invasive diseases (Facklam, 2002). Streptococcal infection in the form of scarlet fever has been described since centuries. Epidemics of both benign and fatal disease were reported, including an epidemic in the American colonies in 1736, which resulted in the death of nearly 4000 people. In the 19th century the lethality of GAS infections dramatically increased in both Europe and North Americ (Baxter and McChesney, 2000; Stevens et al., 1989; Katz and Morens, 1992). The most common infections caused by $S$. pyogenes are grouped as the non- invasive streptococcal disease upper respiratory tract (pharyngitis) and skin (impetigo) infections (Mascini et al., 1999; Stanley et al., 1995; Sneath et al., 1986; Efstratiou et al., 1994; Ross, 1990; Stevens, 1992). Invasive soft tissue infection (Symptoms include pain at the site of infection, fever, malaise, nausea, vomiting and diarrhea, dizziness, confusion and a flat rash over large parts of the body). (Mascini et al., 1999; Stanley et al., 1995; Sneath et al., 1986; Efstratiou et al., 1994; Ross, 1990; Stevens, 1992) and non-suppurative sequelae 'Two serious diseases may develop as separate to S.pyogenes infections, Rheumatic fever and Acute glomerulonephritis. (Mascini et al., 1999; Stanley et al., 1995; Sneath et al., 1986; Efstraitiou et al., 1994; Ross, 1990; Stevens, 1992).

The various virulence factors are involved in adherence of S.pyogenes (Bernnet et al., 1998), M proteins (Gregory et al., 2002),
Capsule and $\mathrm{C} 5 \mathrm{a}$ peptidase (Dale et al., 1996), Steptolysins (Kilian, 1998), Pyrogenic exotoxins (Berner et al., 2000), Hyaluronidase, Streptokinase, Lipoproteinase and Deoxyrihonuclease (Kilian, 1998). In the present study a total of 129 samples were subjected for occurrence of S.pyogenes and we have recorded 51 S.pyogenes isolates. The incidence of S.pyogenes was highest in sputum, pus and throat swab. The incidence in sputum $(60 \%)$ and throat swab (48\%) allow us to speculate that pharyngitis might be endemic to the Gulbarga region. The emergence of $S$. pyogenes as a resurgent pathogen is because of its ability to cause a variety of diseases and also because of the increase in the trends in antibiotic resistance worldwide (Holmstrom et al., 1990; Seppala et al., 1992; Weiss et al., 1997). In the present study the antibiogram of the $S$. pyogenes isolates was studied using eleven antibiotics including those which are routinely prescribed as a treatment model for $S$. pyogenes infection in general and pharyngitis, sore throat in particular. Bacitracin sensitivity was used as preliminary diagnostic aid and was found to be satisfactory. All the isolates have shown resistance to all the antibiotics in varying degree 42 out of the 51 isolates $(82.35 \%)$ showed resistance to erythromycin, the drug of choice to penicillin allergic patients. The results indicate a vast difference compared to earlier reports (Holmstrom et al., 1990; Seppala et al., 1992; Weiss et al, 1997). Isolates showing resistance to 6 of the antibiotics. Three isolates $(5.88 \%)$ showed resistance to 8 antibiotics out of the total 11 . However, none of the isolates was resistant to all the 11 antibiotics used.

The present investigation was undertaken with a main objective of studying the epidemiology of Streptococcus pyogenes strains from socio-economic background 
and characterization of the S.pyogenes and further to study the antibiogram by standard Kirby-bauer disk diffusion method to the commonly used antibiotics.

\section{Materials and Methods}

\section{Sources of sample collection}

The period of study span from January 2004 to August 2004. Patients admitted to Basaveshwara Hospital, KBN Hospital and Teaching Hospital of MRM College Gulbarga, constituted the sources of samples. The samples were taken on the basis of preliminary infection on skin lesion, throat infection, and other pyogenic infections. The samples pus, sputum swabs, from pyogenic sites from clinical patients" were collected in screw cap bottles/ test tubes and transported into the laboratory. The enriched cultures were subjected to preliminary microscopic observation by simple staining, Gram's staining and other Morphological observations." For isolation of the etiological agent Streptococcus pyogenes the Cary Blair medium was used as an enrichment medium (Luechtefield, 1981) and was obtained from hi-Media, Bombay. "For further characterization of Streptococcus pyogenes two more media were used, Blood agar and PYR Agar" (L. Pyrollidiony/acrylamide). Dehydrated media were recomposed as for the manufacturer's specifications (Hi Media).

\section{Bacitracin susceptibility test}

Bacitracin and Taxo AO differentiation disc (0.09-0.05 ji/disc) are used for the presumptive identification and differentiation of Lance field Group A (Streptococcus pyogenes) from other Hemolytic Streptococci. "Maxted showed that group A Streptocci was sensitive to bacitracin might therefore be used as rapid diagnostic agent for group ' $A$ '
Streptococci". The bacitracin Disc Technique was considered to be simplest and most practical for the routine clinical laboratory. The bacitracin sensitivity Triptose Blood agar.

\section{Antibiotic susceptibility testing}

As the Streptococcus pyogenes isolates were subjected to antibiotic susceptibility testing commercially available antibiotic discs (Hi Media, Bombay) (10 - $30 \mathrm{mg}$ ) were used. These antibiotics were selected based on the survey in Gulbarga and also based on the literature that these are choice antibiotics used for the treatment of Streptococcus pyogenes. The method adopted is the KirbyBuer's Agar Disc Diffusion assay (Bauer, 1996). The antibiotic susceptibility discs and the Muller Hinton Agar were obtained from Hi Media Laboratories Mumbai, India. The antibiotics used in the study were ampicillin (10 mg) (30 mg), vancomycin (10 mg) (30 $\mathrm{mg})$ Erythromycin (10 $\mathrm{mg}) \quad(30 \mathrm{mg})$ Chloramphinicol (10 mg) (30 mg) ceftrioxne (10mg) (30 mg) cefaclor (10mg) (30 mg) claritrhomycin $(10 \mathrm{mg})(30 \mathrm{mg})$ clindamycin (10 mg) (30 mg) Levofloaxcin (10mg) (30mg) ofloaxacin (10mg) (30mg) Chephotaxime (10mg)(30mg).

\section{Results and Discussion}

In the present study a total of 129 samples were subjected for occurrence of S.pyogenes and we have recorded 51 S.pyogenes isolates. The sex wise distribution shows males are significantly more susceptible to S.pyogenes infections indicated by $48 \%$ recoveries from males and $20 \%$ from females. The present investigation also made clear that children and young adults below 20 years and 20-40 years are more susceptible to S.pyogenes infection. The incidence of S.pyogenes was highest in the adults of age group 20-40 with an incidence 
of $43.7 \%$. Though the incidence of S.pyogenes in the children's and young adults above 40, the results of age groups 40-50 years are not conclusive as the sample size is small. Samples were collected from various body sites viz., pus, sputum, throat swab, cervical swab and ear discharge. The incidence of S.pyogenes was highest in sputum, pus and throat swab. The incidence in sputum $(60 \%)$ and throat swab (48\%) allow us to speculate that pharyngitis might be endemic to the Gulbarga region. The emergence of $S$. pyogenes as a resurgent pathogen is because of its ability to cause a variety of diseases and also because of the increase in the trends in antibiotic resistance worldwide (Holmstrom et al., 1990; Seppala et al., 1992; Weiss et al., 1997). In the present study the antibiogram of the $S$. pyogenes isolates was studied using eleven antibiotics including those which are routinely prescribed as a treatment model for $S$. pyogenes infection in general and pharyngitis, sore throat in particular. Bacitracin sensitivity was used as preliminary diagnostic aid and was found to be satisfactory. All the isolates have shown resistance to all the antibiotics in varying degree 42 out of the 51 isolates $(82.35 \%)$ showed resistance to erythromycin, the drug of choice to pencillin allergic patients. The results indicate a vast difference compared to earlier reports (Holmstrom et al., 1990; Seppala et al., 1992; Weiss et al., 1997). Though clindamycin is recommended for recurrent $S$. pyogenes pharyngitis (Bisno et al., 2002; Hasenbein et al., 2004) our results show a $82.35 \%$ resistance to clindamycin. S. pyogenes isolates from our study showed a remarkable resistance to ofloxacin $(86.27 \%)$ and cefaclor (86.27\%). Cephotaxime and clarithromycin seem to be suitable alternatives for the patients not responding toerythromycin as the resistance shown by $S$. pyogenes in the current study is only $13.72 \%$ and $21.56 \%$ to cephotaxime and clarithromycin respectively. Multiple antibiotic resistances was also seen among the 51 S.pyogenes isolates. 17 isolates $(33.33 \%)$ showed resistance to 5 Out of the 11 antibiotics used, followed by 14 (27.41\%) isolates showing resistance to 6 of the antibiotics. Three isolates (5.88\%) showed resistance to 8 antibiotics out of the total 11. However, none of the isolates was resistant to all the 11 antibiotics used. During the period from January 2004 to August 2004, 129 clinical samples were collected, from these a total of 51 strains of S.pyogenes have been isolated from the patients admitted to Basveshwar hospital, M.R.M collage and K.B.N hospital, infected with pyogenic infections. Five types of sources of samples were used pus, sputum, cervical swab, throat swab, and ear discharge. The isolates were further characterized and confirmed by microscopic, cultural and biochemical investigations and bacitracin sensitivity tests. Further, antibiogram pattern of the $S$. pyogenes isolates to some of the most commonly employed antibiotics were also investigated.

"Out of the 129 samples screened, $S$. pyogenes was isolated from 51 samples indicating that $39.53 \%$ of pyogenic infections are being caused by $S$. pyogenes (Table-2 and Fig. 1). The incidence of $S$. pyogenes was found to be very high among males than females. Out of the 89 samples collected from males, 43 yielded $S$. pyogenes, indicating an incidence of $48.31 \%$. While in the case of females out of 40 samples only 8 samples showed the presence of $S$. pyogenes indicating an incidence of only $20 \%$. The breakup of $S$. pyogenes isolates from different samples is presented in Table-3 and Fig.2. Highest incidence has been found to be in sputum where in 15 samples out of 25 yielded $S$. pyogenes indicating an incidence of $60.0 \%$. 
The incidence was also high in pus $(50 \%)$ and throat swab (48\%) samples. While the same was very low in cervical swabs $(10 \%)$ and ear discharge (11\%). The age wise distribution of $S$. pyogenes incidence is depicted in Table-4 and Fig. 3. The results indicated highest incidence among young and the middle aged people less than 50 years of age. The patients in the age group of 20-40 years yielded the highest incidence of $50 \%$. While the age group 40-50 indicated an incidence of $43.7 \%$ followed by the age group <20 years showing $40 \%$ Incidence of $S$. pyogenes. The incidence was very low in individuals above 50 years of age. The age group 50-60\% indicated an incidence of only $11 \%$, and in the patients above 60 years $S$. pyogenes was present in $20 \%$ of the cases.

\section{Antibiotic susceptibility testing}

The results of the susceptibility of the 51 isolates of $S$. pyogenes to the 11 most commonly prescribed antibiotics for pyogenic infections are represented, The antibiogram pattern indicated an alarming situation, in that among " $S$. pyogenes isolates were resistant to multiple antibiotics all the 51 isolates were resistant atleast to three antibiotics.

Table.2 Sex wise Incidence of S.pyogenes

\begin{tabular}{|c|c|c|c|}
\hline Sex & $\begin{array}{c}\text { No. of } \\
\text { Samples }\end{array}$ & No. of S.pyogenes isolates & $\begin{array}{c}\text { \% of } \\
\text { incidence }\end{array}$ \\
\hline Male & 89 & 43 & 48.31 \\
\hline Female & 40 & 8 & 20 \\
\hline Total & $\mathbf{1 2 9}$ & $\mathbf{5 1}$ & $\mathbf{3 9 . 5 3}$ \\
\hline
\end{tabular}

Table.3 Incidence of S.pyogenes in different samples

\begin{tabular}{|l|c|c|c|}
\hline Sample Collected & No. of Samples & $\begin{array}{c}\text { No. of S.pyogenes } \\
\text { isolates }\end{array}$ & $\begin{array}{c}\text { \% of } \\
\text { incidence }\end{array}$ \\
\hline Pus & 40 & 20 & 50.0 \\
\hline Sputum & 25 & 15 & 60.0 \\
\hline Throat swab & 25 & 12 & 48.0 \\
\hline Cervical swab & 20 & 2 & 10.0 \\
\hline Ear discharge & 19 & 2 & 11.0 \\
\hline \multicolumn{1}{|c|}{ Total } & $\mathbf{1 2 9}$ & $\mathbf{5 1}$ & $\mathbf{3 9 . 5 0}$ \\
\hline
\end{tabular}


Table.4 Age-wise incidence of S.pyogenes

\begin{tabular}{|l|c|c|c|}
\hline Sample Collected & No. of Samples & $\begin{array}{c}\text { No. of S.pyogenes } \\
\text { isolates }\end{array}$ & $\begin{array}{c}\text { \% of } \\
\text { incidence }\end{array}$ \\
\hline$<20$ & 25 & 10 & 40.0 \\
\hline $20-40$ & 60 & 30 & 50.0 \\
\hline $40-50$ & 16 & 7 & 43.7 \\
\hline $50-60$ & 18 & 2 & 11.0 \\
\hline$>60 \quad$ Total & 10 & 2 & 20.0 \\
\hline & $\mathbf{1 2 9}$ & $\mathbf{5 1}$ & $\mathbf{3 9 . 5 0}$ \\
\hline
\end{tabular}

Table.6 Incidence of multiple antibiotic resistances among S.pyogenes

\begin{tabular}{|c|c|c|}
\hline No. of Antibiotics & Isolates shown V & \% of Resistant \\
\hline 3 & 5 & 9.80 \\
\hline 4 & 4 & 7.84 \\
\hline 5 & 17 & 33.33 \\
\hline 6 & 14 & 27.45 \\
\hline 7 & 8 & 15.69 \\
\hline 8 & 3 & 5.88 \\
\hline Total & $\mathbf{5 1}$ & $\mathbf{1 0 0 . 0 0}$ \\
\hline
\end{tabular}

Table.7 Incidence of antibiotic resistance among S.pyogenes isolates

\begin{tabular}{|l|c|c|c|}
\hline \multicolumn{1}{|c|}{ Antibiotics } & $\begin{array}{c}\text { No. of } \\
\text { Resistivity }\end{array}$ & $\begin{array}{c}\text { No. of } \\
\text { Sensitivity }\end{array}$ & $\begin{array}{c}\text { \% of } \\
\text { Resistant }\end{array}$ \\
\hline Ampicillin & 15 & 36 & 29.41 \\
\hline Vancomycin & 40 & 11 & 78.43 \\
\hline Erythromycin & 42 & 9 & 82.35 \\
\hline Chloroamphenicol & 14 & 37 & 27.45 \\
\hline Ceftriame & 21 & 30 & 41.17 \\
\hline Cefaclor & 44 & 7 & 86.27 \\
\hline Clarithromycin & 11 & 40 & 21.56 \\
\hline Clindamycin & 42 & 9 & 82.35 \\
\hline Levofloxacin & 15 & 36 & 29.41 \\
\hline Cephotaxime & 7 & 44 & 13.72 \\
\hline Ofloxacin & 44 & 7 & 86.27 \\
\hline
\end{tabular}


Fig.1 Sex wise incidence of S. pyogenes

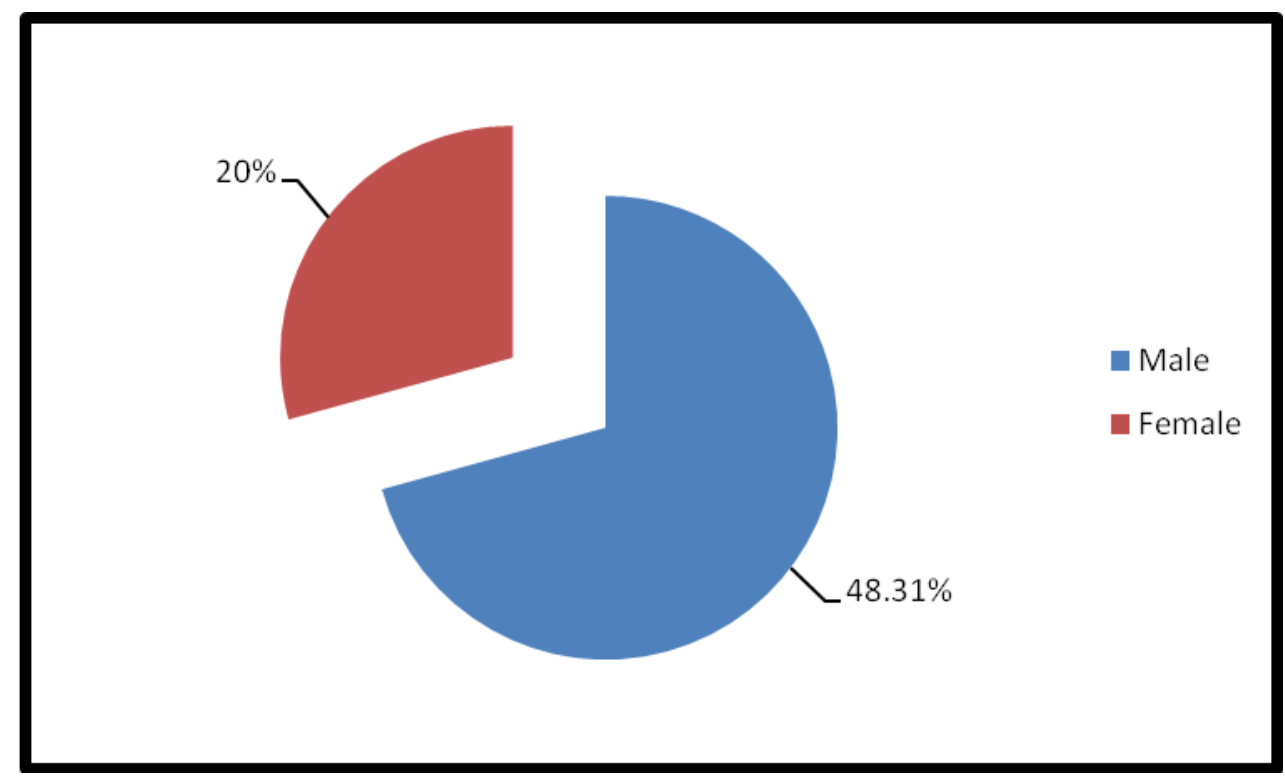

Fig.2 Incidence of S.pyogenes in different samples

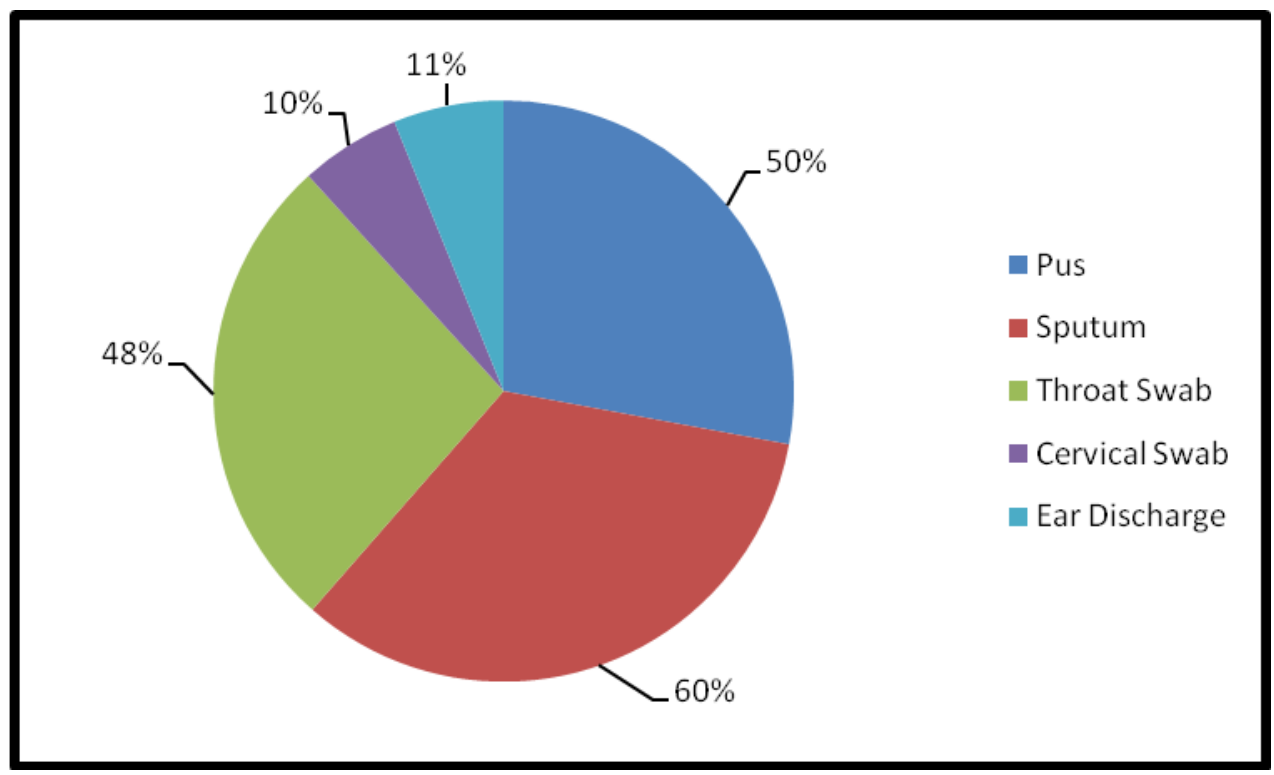


Fig.3 Age wise incidence of S.pyogenes.

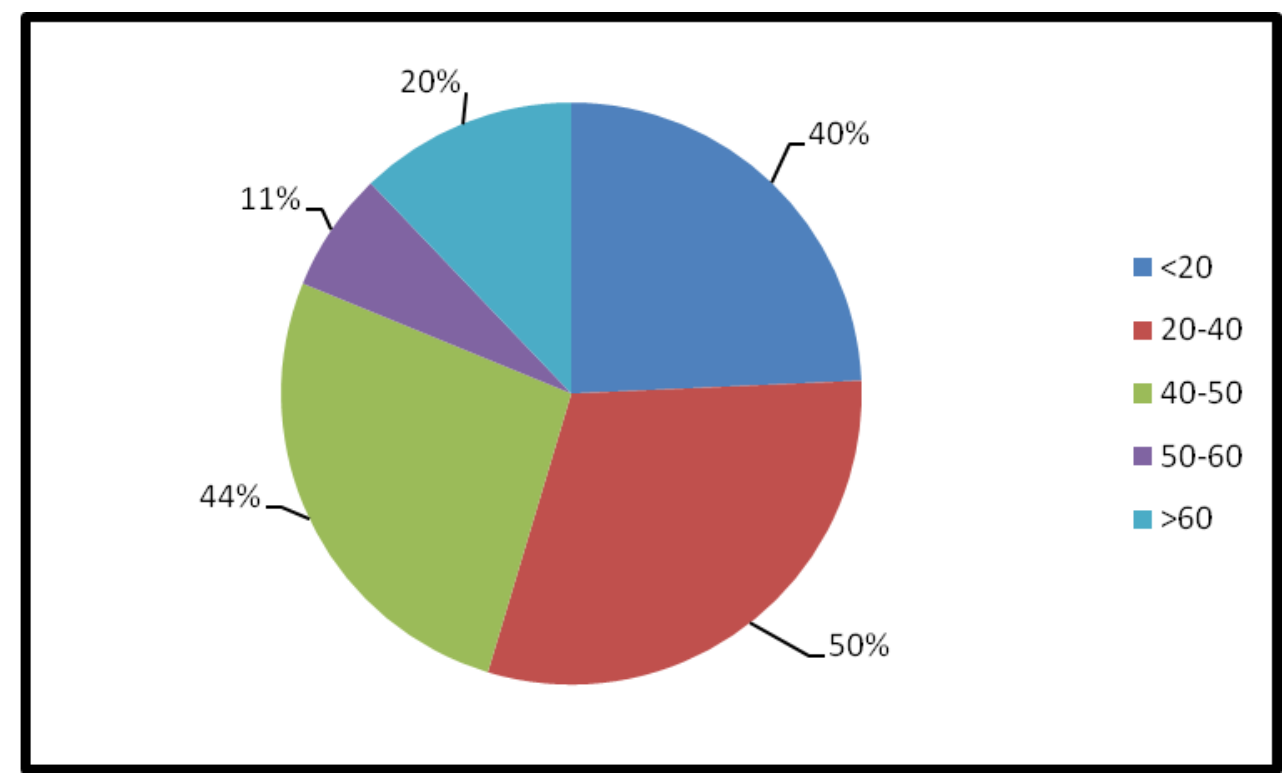

The multiple resistances ranged from 3 to 8 antibiotics. Among the 51 isolates, as many as $17(33 \%)$ were resistant to 5 antibiotics and $14(27 \%)$ to 6 six antibiotics (Table-6). Among the 11 antibiotics, the highest incidence of resistance $(86.27 \%)$ among S.pyogenes isolates was observed against Cefaclor and Ofloxacin and $82.35 \%$ resistance against erythromycin and clindamycin. Resistance also was very high (78.43\%) against vancomycin. However, in respect of other antibiotics low $(13.72 \%)$ to moderate $(41.17 \%)$ resistance was observed (Table-7).

In conclusion, the findings in our study were consistent with the view that Streptococcus pyogenes infection in general population should not be neglected. This study highlights the regular screening and the importance of regular surveillance to keep S.pyogenes in check and by treating early with appropriate antibiotics by routine culture and sensitivity and also highlights upcoming drug resistance to the commonly used antibiotics which may be due to injudicious and excessive use of antibiotic therapy without following proper antibiotic policy.

\section{Acknowledgements}

Authors are grateful to Prof. S.M Gaddad, Department of Microbiology, Gulbarga University, Gulbarga.-585016.karnataka state, India. For his valuable suggestions. Another faculty Dr. C.T. Shivananveur Department of microbiology, Gulbarga University, Gulbarga for his valuable suggestions.

\section{References}

Arvand, M., Hoeck, M., Hahn, H., and Wagner. 2000. Antimicrobial resistance in Streptococcus pyogenes: Antimicrobial Chemother., 46: 62(13).

Bennett, W., Carapetis, J.R. and Brown, R. M. 1998. Ability of clinical isolates of group A streptococci to adhere to and invade Hep -2 epitherlial cells "Journal omedicine.47: 899-906.

Berner R, Herdeg S, Gordjani N, and Brandis M.(2000). Streptococcus 
pyogenes meningitis: Eur. J. Pdt., 159: 527-529.

Bisno, A.L. 2001. Acute pharyngitis new J. England, 344: 205-211.

Dale, J.B., Washburn, R.G., Marques, M.B. and Wessels, M.R. 1996. Hyaluronate capsule and surface $M$ protein in resistance to phagocytosis of group A streptococcus, J. Infect., 64: 14951501.

Efstratiou, A. 2000. Group A streptococci. Antimirobial Chemother., 45: 3-12.

Facklam, R. 2002. "What happened to the streptococci: overview of taxonomic and nomenclature changes, J. Clin. Microbiol., 15: 613-630.

Holstron, M., Dressel, D., and Peterson, L. 1990. Highly Reproducible bactericidal activity test results by using a modified national committee for clinical laboratory standards broth macrodilution technique. J. Clin. Micpbiol., 37: 1881-1884.

Kaplan, E.L. 1997. Recent evaluation of antimicrobial resistance in bhemolytic streptococci: Clin. Infect. Dis., 24: 89-92.

KatZ, A.R., Morens, D.M. 1992. "severe streptococcal infections in historical perspective" Clin. Infect. Dis., 14: 298-307.

Killian, V.L., Chieffi, L.N., Jurfest, M.D. 1998. Neonatal streptococcus pyogenes meningitis and Sagittal Sinus thrombosis: Case Report, Arq. Neuropsiquiatr., 56: 829-832.
Massell, B.F., chute, C.G., Walker, A.M., Kurland, G.S. 1998. Penicillin and the marked decrease in morbidity and mortality from rheumatic fever in the united states: New England J. Med., 318: 280-6.

Maxted, W.R., Widdowson, J.P., Fraser, C., A.M., Ball, L.C., and Bassett, K.C.J. 1973. The use of serum opacity reaction in the typing of gourp A streptococci, J Med., 6: 83-90.

National committee for Clinical Laboratory Standards: Methods for dilution antimicrobial susceptibility tests for bacteria that grow aerobically. Approved standards M7-A4. NCCLS, Wayne, PA (1997) differentiation of human and other groups of hemolytic streptococci. J. Exp. Med., 57: 571595.

Stevens, D.L., Tanner, M.H., Winship, J. 1989. Severe group A streptococcal infections associated with a toxic shock - like syndrome and scarlet fever toxin A. New J. England, 317: 17.

Weiss, K., Azavedo, J., Restieri, C., Galameau, L.A., Gourdeau, M., Harvey, P. 2001. Phenotypic and genotypic characterization of macrolide-resistance group a Streptococcus strains in the province of Quebec, canada. J. Antimicrob. Chemother., 47: 345-8.

\section{How to cite this article:}

Sanjay Rathod, Muzaheed, Venkat M. Shinde, H.P. Jai Shanker Pillai. 2016. Epidemiology of Streptococcus pyogenes in Pyogenic Infections in Gulbarga, India. Int.J.Curr.Microbiol.App.Sci. 5(10): 1030-1038. doi: http://dx.doi.org/10.20546/ijcmas.2016.510.109 